\title{
A System for Combined Three-Dimensional Morphological and Molecular Analysis of Thick Tissue Specimens
}

Rodrigo Fernandez-Gonzalez, Arthur Jones, Enrique Garcia-Rodriguez, Ping Yuan Chen, Adam Idica, Stephen J. Lockett, Mary Helen Barcellos-Hoff, Carlos Ortiz-de-Solorzano

\author{
Life Sciences Division \\ Lawrence Berkeley National Laboratory \\ 1 Cyclotron Road, \\ Berkeley, CA 94720
}

Running title: 3D reconstruction from sections

Corresponding Author:

Carlos Ortiz de Solorzano, Ph.D.

Ms 84-171

Lawrence Berkeley National Laboratory

1 Cyclotron Road,

Berkeley, CA 94720

Ph: (510) 486-4923

Email: $\underline{\text { CODeSolorzano@lbl.gov }}$

Key words: Computer Assisted Microscopy, 3D Reconstruction, Assited Histo-pathology, JAVA. 


\begin{abstract}
We present a new system for simultaneous morphological and molecular analysis of thick tissue samples. The system is composed of a computer assisted microscope and a JAVAbased image display, analysis and visualization program that allows acquisition, annotation, meaningful storage, three-dimensional reconstruction and analysis of structures of interest in thick sectioned tissue specimens. We describe the system in detail and illustrate its use by imaging, reconstructing and analyzing two complete tissue blocks which were differently processed and stained. One block was obtained from a ductal carcinoma in situ (DCIS) lumpectomy specimen and stained alternatively with Hematoxilyn and Eosin (H\&E), and with a counterstain and fluorescence in situ hybridization (FISH) to the ERB-B2 gene. The second block contained a fully sectioned mammary gland of a mouse, stained for Histology with H\&E. We show how the system greatly reduces the amount of interaction required for the acquisition and analysis and is therefore suitable for studies that require morphologically driven, wide scale (e.g. whole gland) analysis of complex tissue samples or cultures.
\end{abstract}




\section{Introduction}

Understanding complex biological systems requires tissue-level integration of information from multiple sources: molecular, physiological, anatomical, etc. However, none of the existing analytical methods in Biology provides the required level of integration, in that they either don't account for intercellular variation (RFLP, Southern blots, microarray technologies, etc.), or they do it at the expense of tissue integrity (e.g. Flow Cytometry).

Image-based Cytometry (IC) can provide molecular or genetic information (e.g. by using fluorescence in situ hybridization -FISH- or immunohistochemistry -IHQ-), in fixed cells within their native morphological tissue context. Volumetric, 3D morphological information can be obtained using confocal laser scanning microscopy (CLSM). However, only relatively thin tissue sections $(<100 \mu \mathrm{m})$ can be studied, due to light scattering and refractive index mismatch problems that occur when imaging deeper in the tissue, and because of practical limitations of effectively staining thicker sections by IHQ or FISH.

When analysis and integration of molecular and morphological information at high resolution is aimed on a bigger scale (e.g. tissue or a small gland), the only existing approach requires sectioning the tissue, followed by both histological and molecular staining of consecutive tissue sections. The analysis is normally performed by visual inspection of the sections under the microscope. This approach greatly limits the extent and accuracy of the analysis, due to the difficulty that our visual system finds when composing (extrapolating) meaningful $3 D$ information from a series of $2 D$ sections. Since only a few colors (2-4) can be discriminated, both in bright field and fluorescence microscopy, along with other practical problems related to multicolor IHQ or $(\mathrm{F}) \mathrm{ISH}$, we have to do the histological and the molecular 
staining on different, alternative, sections. This further complicates the visual analysis and integration of molecular and morphological information.

To overcome these problems, we have developed a three-dimensional microscopy system that integrates computer analysis and visualization tools. These tools automate or greatly reduce the amount of interaction required for the acquisition, reconstruction and morphologically directed analysis of thick tissue samples. Our system can be used to reconstruct tissue structures and to quantitatively measure the presence and spatial distribution of different molecular elements (e.g. genes, RNA's, proteins) in their intact cellular environment. This tool is currently being used to study breast cancer, where heterogeneity and threedimensionality are at the very base of both disease initiation and clonal progression.

Our system encapsulates a three-dimensional visualization system and an image analysis system. The application was developed using a distributed architecture (client-server model), and Java for writing the graphical user interface (GUI) so that it can run remotely on any computer platform. The system allows acquiring and registering low magnification (e.g. 1 pixel = $5 \mu \mathrm{m}$ ) conventional (bright field or fluorescence) images of entire tissue sections. It can also create a virtual 3D reconstruction of the tissue structures, from which new areas of interest can be revisited or reacquired at high resolution (e.g. 1 pixel $=0.5 \mu \mathrm{m}$ ) and automatically analyzed.

Although there are existing commercial and non-commercial software packages which can separately perform some of those functions, the integration of all of them (multiresolution, multicolor acquisition of multifield fluorescence microscopy images; reconstruction of structures on interest in 3D; molecular and morphological 3D analyses; content-based image and data 
storage and retrieval system through the use of "Cases", or series of consecutive images and data belonging to a given tissue) on a single platform makes of our system a very powerful tool.

A comparison to similar systems shows that many of them offer a set of independent programs running under different interfaces $(I M O D$, Boulder Laboratory for 3-Dimensional Fine Structure), but not a common platform that integrates all of them. Most of these systems are not designed to run on multiplatform environments (VIDA, University of lowa.; Trace, Boston University), some others have special hardware requirements for real time rendering (VoxBlast, Vaytek Inc.) and many lack a proper image management system (Imaris, Bitplane AG). In this paper we describe our system and we illustrate its use by presenting the reconstruction of a mouse mammary gland and a tumor biopsy of a patient with ductal carcinoma in situ (DCIS) of the breast.

\section{Materials and Methods}

\section{System Description}

The system is controlled by a client-server application, as shown in Figure 1. The server is a C language application that runs on a computer (Dell Inspiron, running Solaris 7 for Intel) connected to an Axioplan (Zeiss Inc., Germany) microscope. The server actuates all the moving parts of the microscope: motorized scanning stage, excitation filter wheel and arc-lamp blocking shutter (Ludl Electronic Products Ltd., Hawthorne, NY). It controls the CCD Microlmager camera (Xillix Techonologies Corp., Richmond, British Columbia, Canada) as well. The server can perform basic operations, such as acquiring and storing images, setting the exposure time of the CCD, moving the stage and operating the filter wheel. In addition, it has been programmed to offer more complex functions, such as automatically focusing the microscope or acquiring multiple field-of-view images. To do this, the server receives each order and divides it 
into a set of simple actions. For instance, to acquire a multiple field-of-view image, the server asks for the coordinates of the vertices of the area to be acquired and then automatically performs the required sequence of stage movements and camera acquisitions. The output is a mosaic-like image of the area. The server can do multi-color acquisition in fluorescence and bright field, by performing consecutive acquisition using different excitation filters and multi band emission filters.

\section{Description of the client application}

The client, (R3D2) is connected to the server through UNIX sockets, which are the standard for Internet-based communications. It can send requests to the server from any computer connected to the Internet. To obtain the maximum benefit from this, R3D2 has been written in JAVA (v.1.2), so that it can be executed on many different computer platforms.

Figure 2 shows the R3D2's complete Graphical User Interface (GUI). The interface is divided in two distinguishable parts. One -rightmost vertical panel in Figure 2- provides connections to the server and allows the user to request its services through a user-friendly interface. The available actions can be classified in two groups:

\section{Basic control operations:}

These include all the simple -atomic- operations provided by the server, such as setting the objective lens, changing the excitation filter (fluorescence), setting the exposure time of the camera, moving the stage to the absolute origin of coordinates relative to which all measurements are taken, opening/closing the arc lamp blocking shutter, and acquiring a single image using the current microscope settings. R3D2 receives the image from the server and displays it, both complete -zoomed out- as well as partially -in its original resolution-. Only a small part of the full-resolution image can be displayed at full resolution. The zoomed area can 
be interactively selected by moving a window on the complete version of the image (Figure 3). Images can be saved both in ICS (Dean et al. 1990) and JPEG format. When images are saved as ICS, all the acquisition parameters (objective, filter, location of the image on the slide are, etc.) are stored in the ICS header file. JPEG format-compressed or not- can be used as an alternative format when the user does not plan any future analysis of the images, and images are stored for exchange, document creation or web publishing.

\section{Complex Operations:}

These operations combine multiple atomic operations to provide the following functionality:

- Autofocus: Automatically focuses the microscope by taking a series of images at different positions in the $Z$ axis (step size $=0.50 \mu \mathrm{m}$ for low magnification images, $0.25 \mu \mathrm{m}$ for high magnification images) and determining the best-focused image of the series. Blur, due to out of focus light, reduces image contrast, which can be detected using several functions. Based on several comparisons described in the existing literature (Groen et al. 1985, Firestone et al. 1991, Santos et al. 1997), we selected an autocorrelation-based function introduced by Vollath (1987).

- Scan: Acquires multiple field of view images. The system displays a dialogue-panel where the user can specify the filter(s) to be used (in fluorescence microscopy), exposure time(s) and the limits of the area to acquire. The limits can be defined by its coordinates (when known) or manually, by moving the microscope to the upper, lower, rightmost and leftmost points of the area. 
- Revisit Point: When the user clicks on a point of the image of a previously acquired complete or partial tissue section, the server moves the stage to that location on the slide and takes an image using the current values of objective, filter and exposure time.

- Revisit Area: When using this option, the user is asked to draw a rectangle on one image of a previously acquired section. The selected area will be then acquired with the microscope settings provided by the user. Multicolor area acquisition is an option as it is for scanning complete sections.

The second part of the interface (two left vertical panels in Figure 2) expands the system capabilities, by allowing creating and handling sets of related images, which we call Cases. A Case is a sequence of low-magnification images of complete tissue sections taken from a tissue block, along with all the areas re-visited on them at higher resolution and with different filters, plus the results of analysis performed on them if any. The image files that make up a Case are specially labeled for convenience. The user can:

- Annotate the Cases, by marking and/or delineating structures of interest and linking them within and between consecutive sections. The user can add textual annotations (Text), ductal structure identifiers (with a unique number that identifies them within the section Duct) and forms that delineate irregularly shaped ducts or other structures (Shapes). In addition to this, ductal marks can be connected within the same section or in different sections (Connected Ducts), and corresponding shapes in different sections can be grouped (Groups). 
- Register acquired sections. Before reconstructing a Case in 3D, all its sections must be registered to ensure proper alignment of the elements that will be later reconstructed. For that, we calculate the Rigid-Body Transform that provides the optimum rotation and translation between each pair of sections. The Transform is calculated from three pairs of points interactively marked by the user on each pair of images to be registered. Once the points have been marked, the software calculates the rotation and translation $\left(\theta, t_{x}, t_{y}\right)$ needed to minimize the sum of the squared distances between all three pairs of corresponding points, thus aligning both images. The results are stored in the second image. This method is very accurate when the pairs of points are spread all along the sections. Reasons for small errors are imprecise mouse interaction, stretching and/or compression of the tissue, and the fact that some structures used to select pairs of corresponding points might not be perpendicular to the sections.

- Reconstruct Cases. Our system reconstructs the tissue structures by rendering the user annotations in 3D. Besides the obvious advantage of volumetric tissue structure visualization, the $3 \mathrm{D}$ rendering is linked to the microscope for revisiting, and to the original images and their analysis for display of the images and analysis results, as will be described later. The $3 \mathrm{D}$ reconstruction part of the software has been developed using Java3D (v.1.2.1.) This is an application-programming interface (API) for 3D in Java. After asking the user for the range of sections to render, the system converts the coordinates of all the markings in that range of sections from two-dimensional to three-dimensional values. The section number and thickness, along with the distance between sections, determines the $Z$ -depth- coordinate. Then a 3D scene is built using several geometric shapes to represent the different markings. Duct markings are rendered as spheres and connected with lines within and between sections. Contour Shapes are rendered as volumes after applying a refined Delaunay triangulation, using the Nuages reconstruction software (INRIA, France, 
http://www-sop.inria.fr/prisme/) (Boissonnat et Geiger 1993). The 3D rendition of the Case is displayed in a new window where the mouse can rotate the scene, zoom in or out or translate the scene in the $X$ and/or $Y$ directions. The 3D window includes a tool bar with options to select elements. By just clicking on one element of the 3D scene, the user can get information about it (location, size, etc.), load the image(s) that contain that element images are displayed in a new image panel- or move the microscope to its location on the slide for re-imaging. Volume selection is handled by JAVA 3D. The selection is performed by tracing a 'virtual ray' from the user's point of view (defined when rendering the Case, normally at a point corresponding approximately to the position of the user's eye) and the point where the user clicks on the screen. The selected volume will be the first object intersected by the ray within the 3D scene. The user can also hide or show all the different elements of the scene, reset it to the default view and change the scale in any of the three dimensions.

- Analyze Cases. All areas selected based on the 3D morphological Reconstruction, can be batch processed upon a user request. This way, only those areas selected based on a particular morphological feature are analyzed, and not all the tissue sections, thus reducing the amount of work required. The analysis is done by streamlining the selected images to a new process running custom-made image analysis routines built on a commercial image processing software (Scilimage, TNO, The Netherlands). At the moment, the image analysis routines can segment counterstained nuclei and detect and quantify FISH probes or punctuate-patterned expressed proteins. The image analysis algorithms for nuclei and signal segmentation have been described elsewhere (Malpica et al 1997, Ortiz de Solorzano et al 1998, Ortiz de Solorzano et al 1999). The results of the analysis can be displayed from the $3 \mathrm{D}$ rendering window, and global measurements can be performed after selecting the volumes. 
An important feature of R3D2 is that all Case-handling and marking functions can be used in parallel to the functions that request microscope actions. Therefore, acquiring a new section can be done in parallel to any other Case related function (e.g. registering already acquired sections or annotating the images). Our implementation of this feature uses Solaris threads. Threads permit executing multiple parallel copies of a program without multiplying resource use. Each thread shares memory and other resources with other threads. R3D2 runs on a main thread and when a microscope-based operation is selected, it launches a new thread that runs on the same memory space as the main one. This scheme guarantees that, in the case of a microscope failure or a socket error, the system will not die abruptly, as only the thread working on the microscope will be affected.

\section{Results}

We will now illustrate the use of our system by showing how two different tissue blocks were imaged and reconstructed. The first one $(\mathrm{HB})$ is a tissue block from the mammary gland of a patient with ductal carcinoma in situ of the breast (DCIS). The second block (MB) is a normal mammary gland of a nuliparus mouse.

\section{Tissue source}

HB. The tissue was part of a breast lumpectomy specimen. After surgery, the specimen was fixed in an alcoholic-formalin solution, and embedded in paraffin per routine at California Pacific Medical Center in 1981. The tissue was originally staged as a T1N0M0 (Stage 1). Several blocks of approximately 1 "x 1 "x $3 \mathrm{~mm}$ were taken from the tissue. The block we used contained DCIS and benign ducts; there was no invasive tumor present. The tumor was positive 
for ERBB2 by immunohistochemistry performed on a $4 \mu \mathrm{m}$ section taken from the top of the block.

MB. Female BALB/c mice were obtained from Simonson (Gilroy, CA) and housed 4 per cage with chow and water ad libitum in a temperature and light controlled facility. Carbon dioxide inhalation was used to kill the animals in accordance with the Association for Assessment and Accreditation of Laboratory Animal Care guidelines and institutional review and approval. The 4th inguinal glands were removed for histology and whole mounts at 10 weeks. The tissue was formalin-fixed and paraffin-embedded.

\section{Tissue preparation}

HB. The human block was sectioned at $4 \mu \mathrm{m}$ thickness. Every 5 th section was collected onto a plus (treated) slide, for a total of 66 sections. From this set, every other section was collected and stained with H\&E (33 sections, $40 \mu \mathrm{m}$ apart). The rest of collected sections were sent to the UCSF Cytogenetics core for FISH (33 sections, $40 \mu \mathrm{m}$ apart), with a probe for the ERBB2 gene, which is amplified in $30 \%$ of carcinomas of the breast. The ERBB2 probe was RMC17P077, a P1 probe. The DNA was extracted and labeled by Nick translation with red CY3 dUTP fluorochrome. In summary, the odd-numbered sections were H\&E stained and the evennumbered sections were DAPI counterstained and processed for FISH.

MB. The mouse gland was paraffin-embedded, H\&E stained and sectioned at $4 \mu \mathrm{m}$. We starting collecting every fifth section (20 $\mu \mathrm{m}$ apart). $80 \mu \mathrm{m}$ into the tissue we switched and 
collected all remaining sections ( $4 \mu \mathrm{m}$ apart), for a total of 24 sections. The collected sections were all placed on glass slides for microscopy and imaging.

\section{Imaging of tissue sections}

HB. Full tissue sections were imaged in bright field (odd numbered sections) (Figure 4A), or fluorescence (even numbered sections) (Figure 4B), using R3D2's Scan option. Fluorescent sections were imaged using a single-band filter block (360 nm excitation) for DAPI.

MB. All sections were imaged in bright field (Figure 4C).

In both blocks, sections were imaged at $10 \mathrm{X}$ with a Fluar (0.5 n.a.) objective lens (Zeiss, Wetzlar, Germany). In order to optimize memory use while keeping enough resolution for histology, images were reduced by a factor of 4 in both $X$ and $Y$ directions, which gave us an effective 2.5X magnification, i.e. a sixteen fold reduction in image size. After image compression, the average image size of the sections was 25 Mbytes in the human block and 10 Mbytes in the mouse. The compression was necessary to speed up image transmission and display.

\section{Creation of Cases}

Two Cases were created, initially empty. The acquired sections were added with a number equal to its section number within the tissue. All sections were manually registered as previously described to ensure proper alignment of the to-be-done annotations. Each section was registered to its previous section, thus aligning all sections with the first H\&E section of the block. 


\section{Tissue Annotation}

HB. All H\&E sections were manually annotated. We marked the centers of the ducts by placing a circular mark (R3D2's Duct tool) in the lumen -when the duct was perpendicular to the image plane- or with a line -when the duct was sectioned longitudinally-. Then we connected the markings within and between consecutive sections using the Connect Ducts option. We also delineated tumor areas using R3D2's Shapes option and grouped their consecutive sections using the Group option. (Figure 5).

MB. The same procedure was followed for the mouse except for the Shapes, which were not used to delineate tumor areas, but the lymph nodes. (Figure 6)

\section{D Reconstruction}

The reconstruction of the Cases, based on the H\&E sections, is shown in Figures 7 (HB) and Figure $8(\mathrm{MB})$. All the user markings are interactive, in that by clicking on them the user can i) obtain positional information, ii) load the part of the original image section(s) containing that marking or iii) revisit the selected marking under the microscope. For the latter the microscope automatically moves to the position of the marking in the tissue, provided that the right slide is on the microscope. In situ tumors and lymph nodes were rendered as volumes in $3 D$, and ducts as lines.

\section{Revisit of Areas of Interest}

HB. Several areas were revisited at high-resolution (40X), with a Plan-Neofluar (1.3 na) oil immersion lens (Zeiss, Wetzlar, Germany). Areas were manually selected having either morphologically normal or abnormal (DCIS) areas. Areas were double-scanned using a Pinkel filter set (Chroma Technologies, Brattleboro, VT), by consecutively imaging while exciting the sample at 360 (DAPI emission from the nuclei) and $572 \mathrm{~nm}$ (CY3 emission from ERBB2 gene). 
All areas were acquired at full resolution and then compressed by a factor of 2 in both $X$ and $Y$ directions for an effective 20X magnification. Areas were manually selected by drawing rectangles on the low-resolution images of the whole fluorescent sections, although they could have been selected from the 3D reconstruction as well. To ensure proper alignment between the areas and the sections, the images of the sections were previously aligned with the microscope slide by calculating the shift between the current location of the slide and its location when the image of the section was originally acquired. This is done by calculating the rigid body transform between three points manually selected in the image of the section and the corresponding points under the microscope. Figure 7 shows the $3 D$ reconstruction of the human Case, incorporating the high-resolution acquired areas, displayed as rectangles. As all other elements in the reconstruction model, the areas can be loaded or revisited by clicking on them (Figure 9). The total number of areas imaged was 160.

\section{Analysis of Areas of Interest}

HB. All 160 areas were segmented overnight using the Analyze Case function. In this particular case, DAPI counterstained nuclei were segmented using a two-step algorithm that first uses an adaptative threshold to separate DNA areas from the background and then applies a Hough-transform + Watershed algorithm to separate clusters of nuclei resulting of the adaptative thresholding. FISH signals were segmented using a TOP-HAT morphological algorithm followed by morphological reconstruction. The FISH segmentation algorithm was applied only to those areas identified as nuclei on the DAPI channel. A detailed description of these methods is out of the scope of this paper and can be found in (Malpica et al 1997, Ortiz de Solorzano et al 1998, Ortiz de Solorzano et al 1999).

The total number of nuclei segmented was above 200,000 . The results of the nuclear and FISH segmentation can be displayed for every area (Figure 9) from the images of the sections they belong to or directly from the 3D rendering window. 


\section{Discussion}

We have presented a new and powerful computer-based system that allows automation of the acquisition, storage and analysis of thick -sectioned- tissue specimens. The system has been described and demonstrated by reconstructing tissue blocks from two tissue sources, processed using different staining and microscopy protocols.

By integrating information from different types of staining, both histological (e.g. H\&E) and molecular (e.g. IHQ or FISH), we allow simultaneous morphological and molecular analysis of the specimens. The molecular analysis is not only simultaneous, but in fact driven by the morphology as the areas acquired and analyzed can be selected directly from the 3D reconstruction of the structures marked on the low-resolution images. This way, the labor intensive task of acquiring and analyzing the images is done semi-automatically -the slides still need to be manually placed on the microscope and the areas to be acquired drawn on the images of the sections-, enormously reducing the time and labor. In fact, large-scale analysis can be done (e.g. whole gland analysis), which would be unthinkable otherwise.

A classical problem of fluorescence microscopy, which limits the number of labeled elements to the number of fluorochromes that can be discriminated from, can be overcome by using single or dual color staining on several adjacent sections, provided that the distance between histological (e.g. H\&E) sections enclosing them allows detection of continuity between the structures of interest.

Future work will involve adding new functionality to the system by augmenting the number of analysis functions provided (e.g. detection of cytoplasmic or extra cellular proteins), and automating the detection of the structures of interest, which at this point is the most time consuming task when reconstructing a Case. Interaction could be further reduced by using an 
automatic slide feeder attached to the microscope, which would eliminate the manual loading of slides for revisiting or acquisition of areas. 


\section{Acknowledgments}

This work is being funded by the Department of Defense Breast Cancer Research Program, project numbers DAMD17-00-1-0306 and DAMD17-00-1-0227. We would like to thank K. Chew and S. Ravani for their help selecting and coordinating tissue sectioning and staining, Drs. G. Maggrane and K. Ewan for their work on the IHQ and FISH staining of the sections, Drs. F. Waldman, K. Chin and J. Gray for their support and advice regarding the selection of the tissue and A. Harris for her help with tissue imaging and annotation. 


\section{References}

Boissonnat JD, and B. Geiger. Three dimensional reconstruction of complex shapes based on the Delaunay triangulation. In: Acharya RS and Goldgof DB editors. Biomedical Image Processing and Biomedical Visualization. SPIE 1905, pp 964-975.

Dean P, Mascio L, Ow D, Sudar D, Mullikin J. 1990. Proposed standard for image cytometry data files. Cytometry 11:561-569.

Firestone L, Cook K, Culp K, Talsania N, Preston K. 1991. Comparison of autofocus methods for automated microscopy. Cytometry 12,:95-206.

Groen F, Young IT, Ligthhart G. 1985. A comparison of different focus functions for use in autofocus algorithms. Cytometry 12:81-91.

Malpica N, Ortiz de Solórzano C, Vaquero JJ, Santos A, Vallcorba I, García-Sagredo JM, del Pozo F. 1997. Applying watershed algorithms to the segmentation of clustered nuclei : Defining strategies for nuclei and background marking. Cytometry 28 :289-297.

Ortiz de Solórzano C, Santos A, Vallcorba I, García-Sagredo JM, del Pozo F. 1998. Automation of FISH Spot counting in interphase nuclei : Statistical Evaluation and Data Correction. Cytometry 31:93-99. 
Ortiz de Solórzano C., García Rodriguez E, Jones A, Sudar D, Pinkel D, Gray JW, Lockett SJ. 1999. Segmentation of Confocal Microscope Images of Cell Nuclei in Thick Tissue Sections. Journal of Microscopy, 193(3):212-226.

Santos A, Ortiz de Solorzano C, Vaquero JJ, Pena JM, Malpica N, del Pozo F. 1997. Evaluation of autofocus functions in molecular cytogenetic analysis. Journal of Microscopy 188(2):264272.

Vollath D. 1987. Automatic focusing by correlative methods. Journal of Microscopy 147: 249288. 


\section{Figure Legends}

Figure 1. Description of the client-server architecture of R3D2

The server runs on a computer with a microscope attached, and provides access to the microscope functions. The client is a JAVA application, which can run on any computer (no particular OS required) connected to the server through the Internet Protocol (IP). The communication between client and server uses UNIX sockets. The client provides user-friendly access to all the microscope functions offered by the server, and allows handling of sets of related images (Cases) for storage, annotation and 3D reconstruction of structures of interest.

Figure 2. R3D2's Graphical User Interface

The GUI is divided in two main parts. The two leftmost panels can display consecutive sections of a Case. It also provides the user with tools for registering sections, annotating the images, connecting structures between sections (e.g. mammary ducts, tumor volumes), and reconstructing the annotated images in 3D. The rightmost panel gives access to the microscope related functions offered by the Server.

Figure 3. Single image acquisition

To acquire a single image, the user clicks on the "Acquire" button, after choosing the appropriate microscope settings: objective lens, excitation filter (in fluorescence) and exposure time of the CCD camera. The "Autofocus" option automatically finds the correct focus plane of the microscope before acquiring an image. The top panel contains the entire image (reduced to fit in the window), while the lower panel contains a zoomed version of the part of the original image selected by the rectangle on the top window. The 
rectangle can be manually moved to look at different parts of the image at its original -fullresolution.

Figure 4. Tissue acquisition

Images of full sections of a tissue block of ductal carcinoma in situ of the breast $(A, B)$ and of a mouse mammary gland $(\mathrm{C})$. Both blocks were paraffin embedded, sectioned and stained for Histology (H\&E, images A and C) or counterstained with DAPI (B). Microscope focusing and image acquisition is completely automated, as described in the text. As for the individual images, the upper panel contains the entire section while the lower contains a zoomed sub-area of it.

Figure 5. Tissue Annotation (Human Tissue)

Two consecutive H\&E stained sections of a human DCIS Case. Only H\&E sections are now being shown. The images show manual annotations, namely ducts and tumor masses. Ducts are connected within and between sections. Tumor contours are drawn and also connected between consecutive sections. Connections between sections are shown by changing line color

of all connected components every time one of them is visited (selected or just traveled over by the mouse pointer).

Figure 6. Tissue annotation (mouse tissue)

Two consecutive H\&E stained sections of the mouse mammary gland used to test the system. Ducts are connected within and between sections. Lymph node countours are drawn and also connected between consecutive sections. Connections between sections are shown by changing line color of all connected components every time one of them is visited (selected or just traveled over by the mouse pointer). 
Figure 7. Tissue Reconstruction (Human Case).

3D reconstruction of the manually annotated DCIS Case. A. Tumor volumes have been surfacerendered to show their three-dimensional shapes. Ducts are identified by spheres and connected by lines within and between sections. Yellow rectangles identify areas that were reacquired at higher magnification. B. Close up view of a set of connected markings corresponding to the same duct. The $3 \mathrm{D}$ reconstruction is fully interactive. It can be rotated, zoomed, and all the elements can be selected to retrieve information about the element, display the original image that contains the element or automatically move the microscope to the selected point.

Figure 8. Tissue Reconstruction (Mouse Case).

3D reconstruction of the H\&E stained, manually annotated mouse Case used to test our system:

(a) H\&E stained. Lymph node volumes have been surface-rendered to show their threedimensional shapes. Ducts are identified by spheres and connected by lines within and between sections.

Figure 9. Revisiting and Analysis of Areas of Interest

Revisited area of the Human DCIS tissue block used to test the system. A) DAPI image of counterstained nuclei, B) CY3 images of FISH with a probe to the erb-b2 gene. Aereas can be acquired and loaded from the images or the sections they belong to or directly from the 3D reconstruction of the tissue. C) Results of the segmentation of nuclei based on the counterstained channel. Each segmented nucleus is colored differently. 
Figures

Figure 1

Java Client

Server Microscope

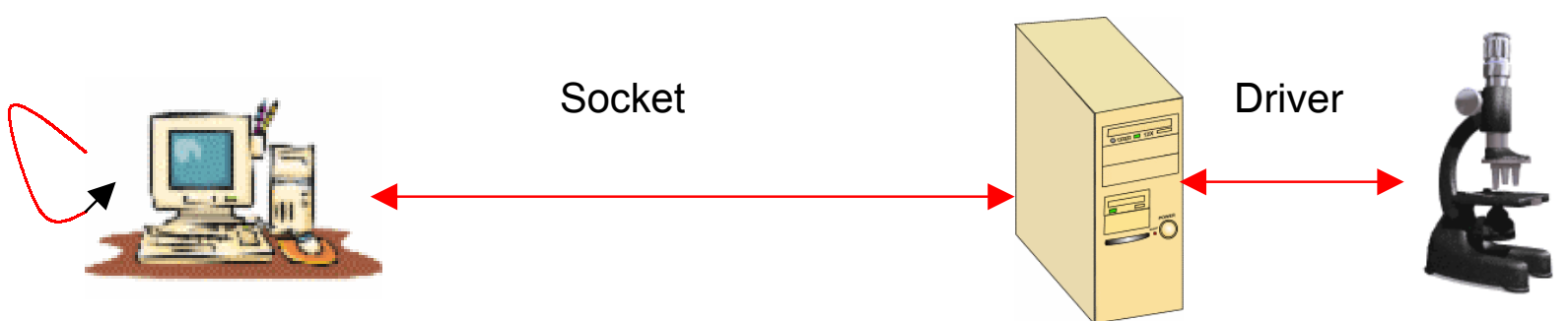


Figure 2

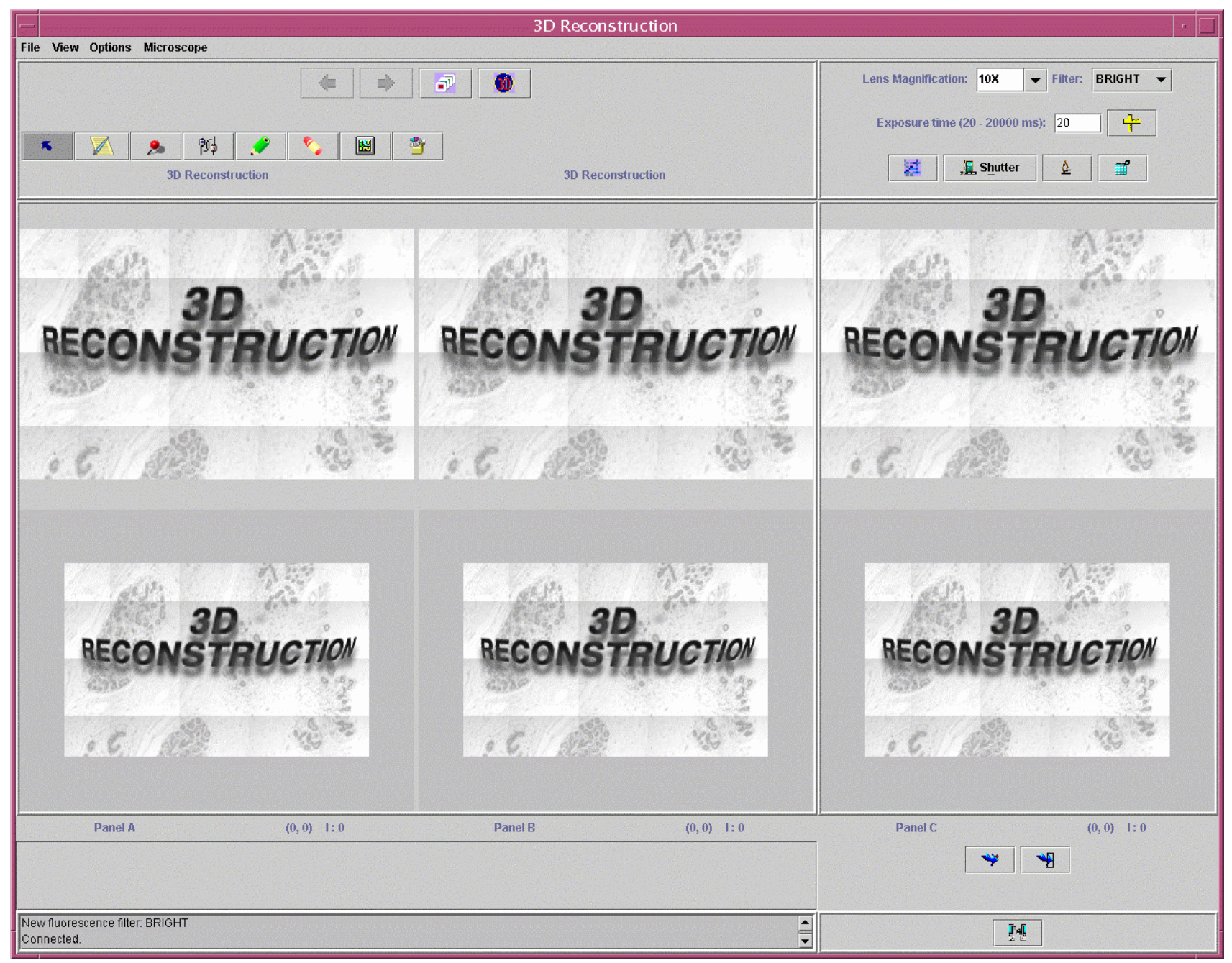


Figure 3

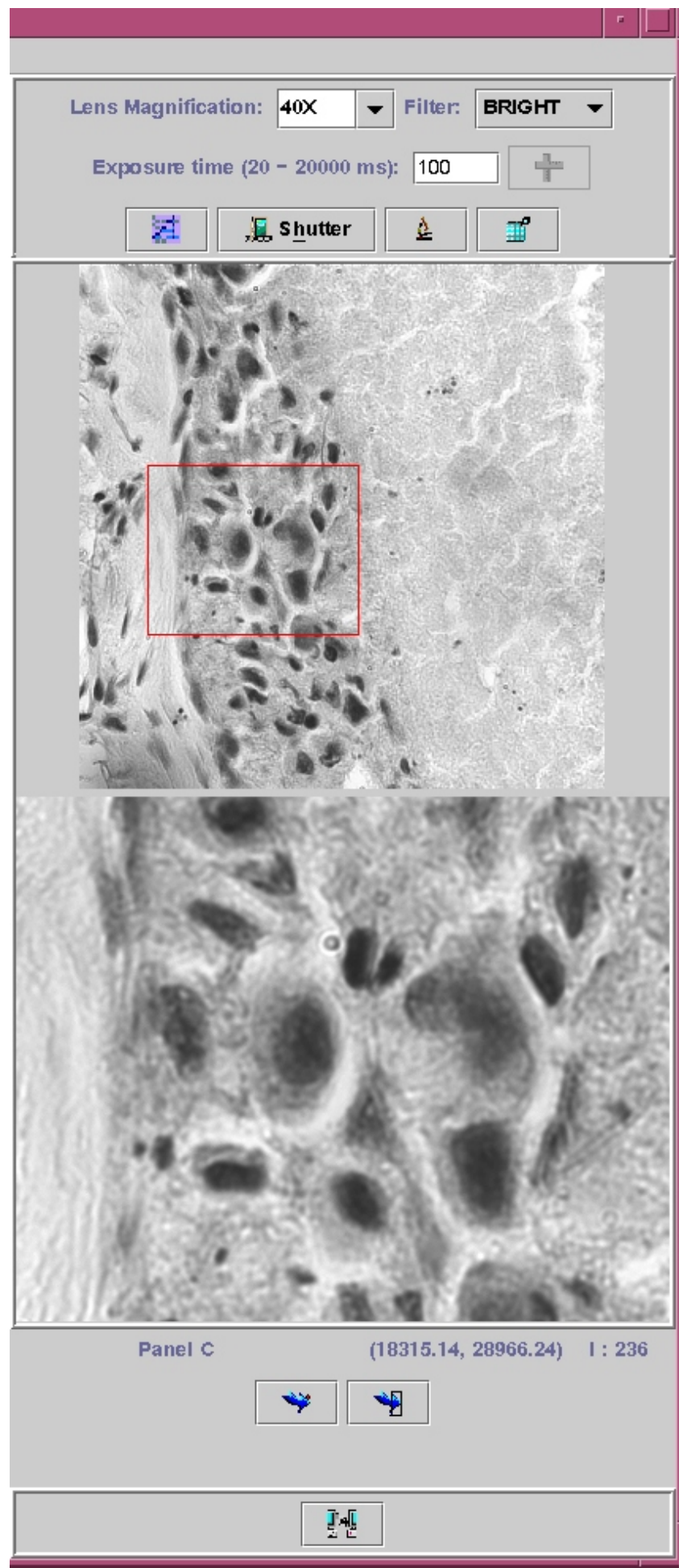




\section{Figure 4}
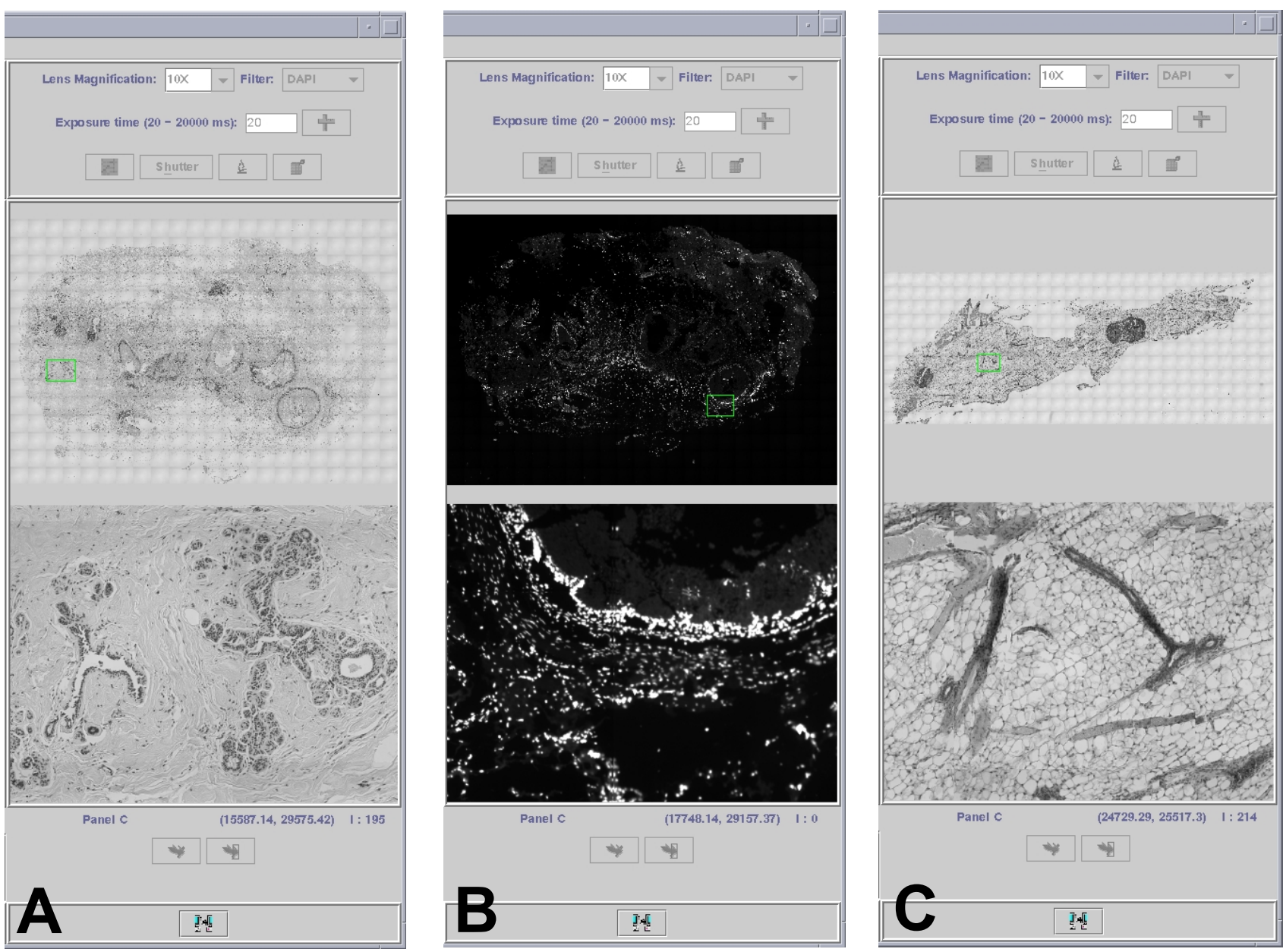
Figure 5

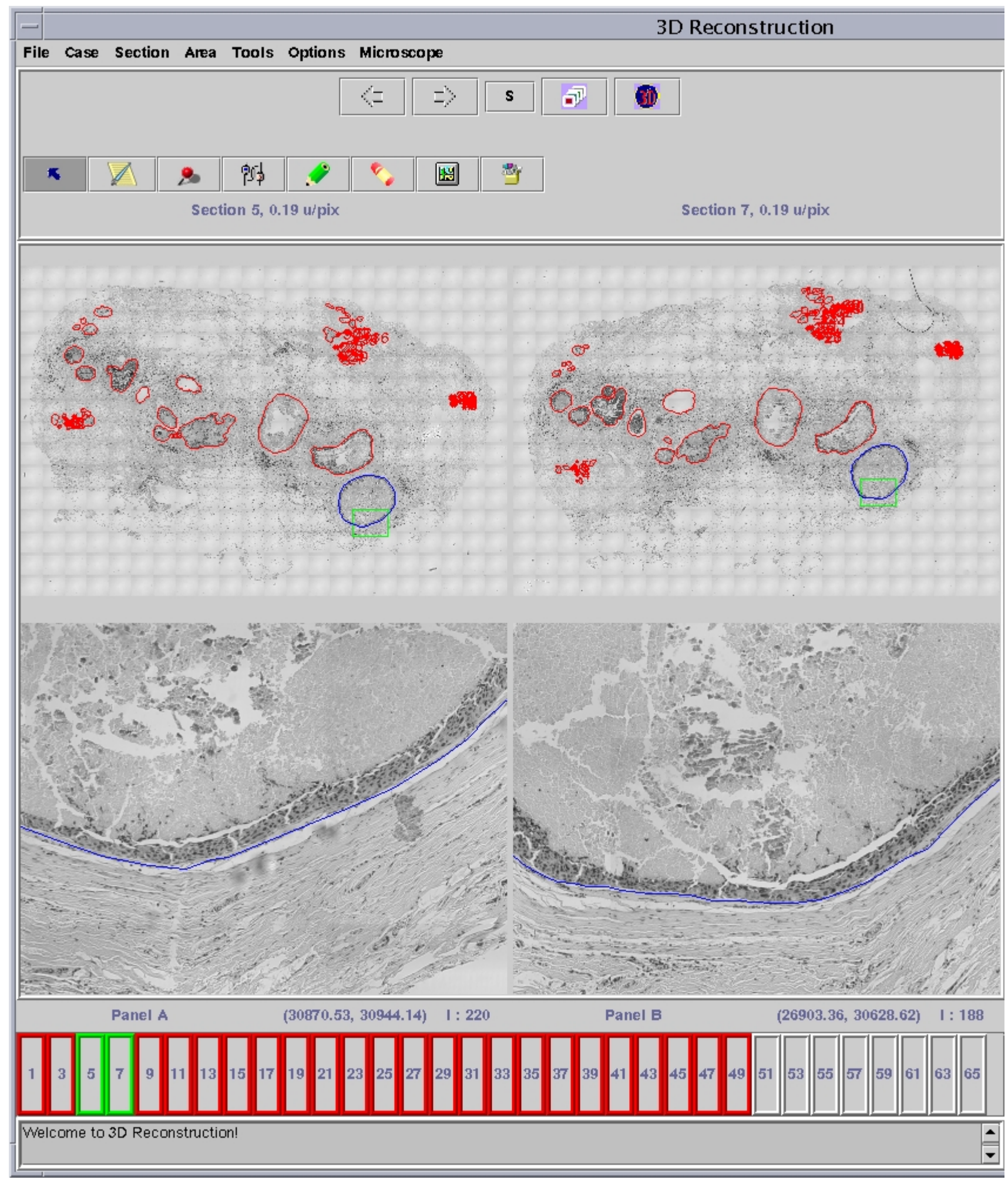


Figure 6

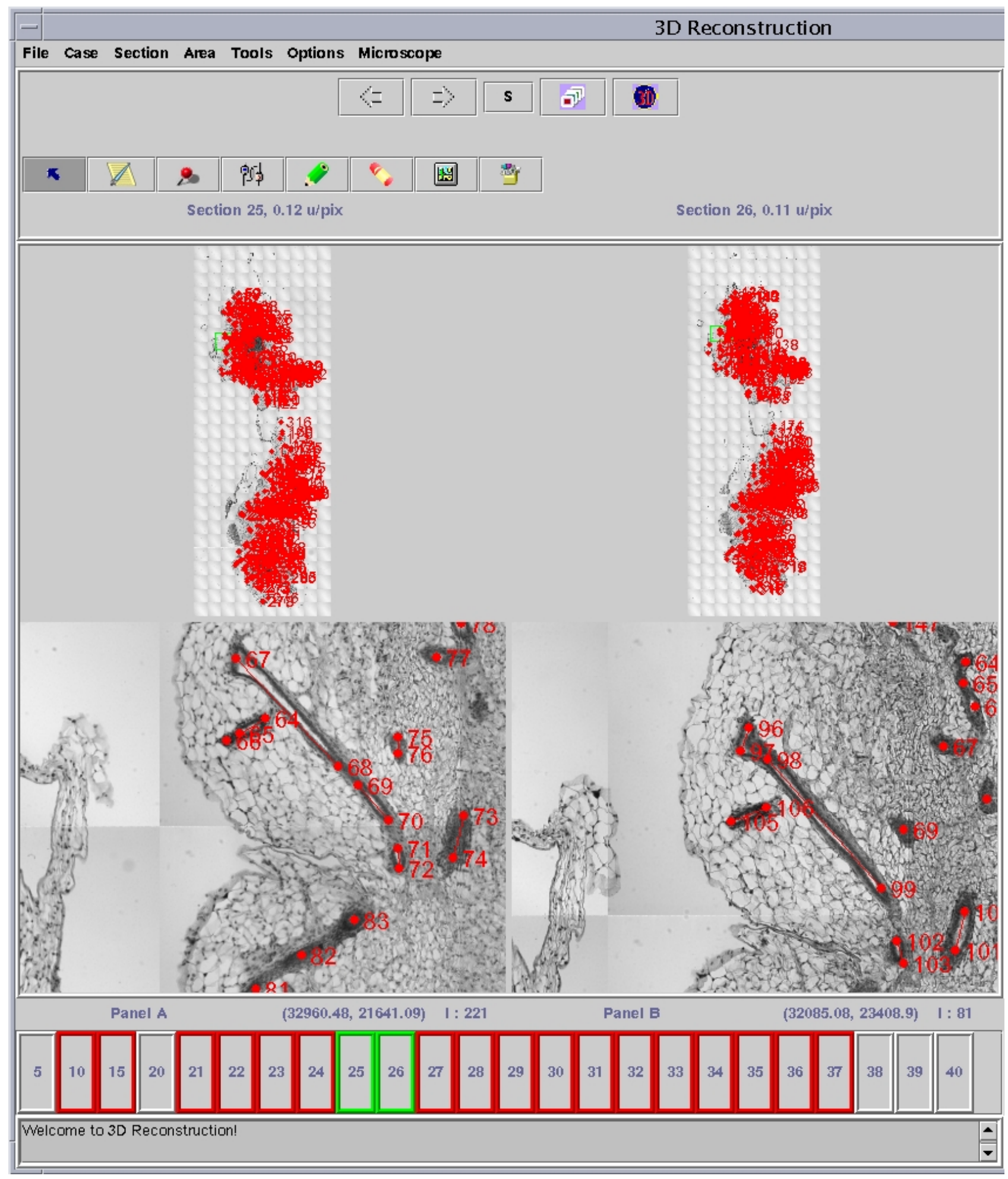




\section{Figure 7}
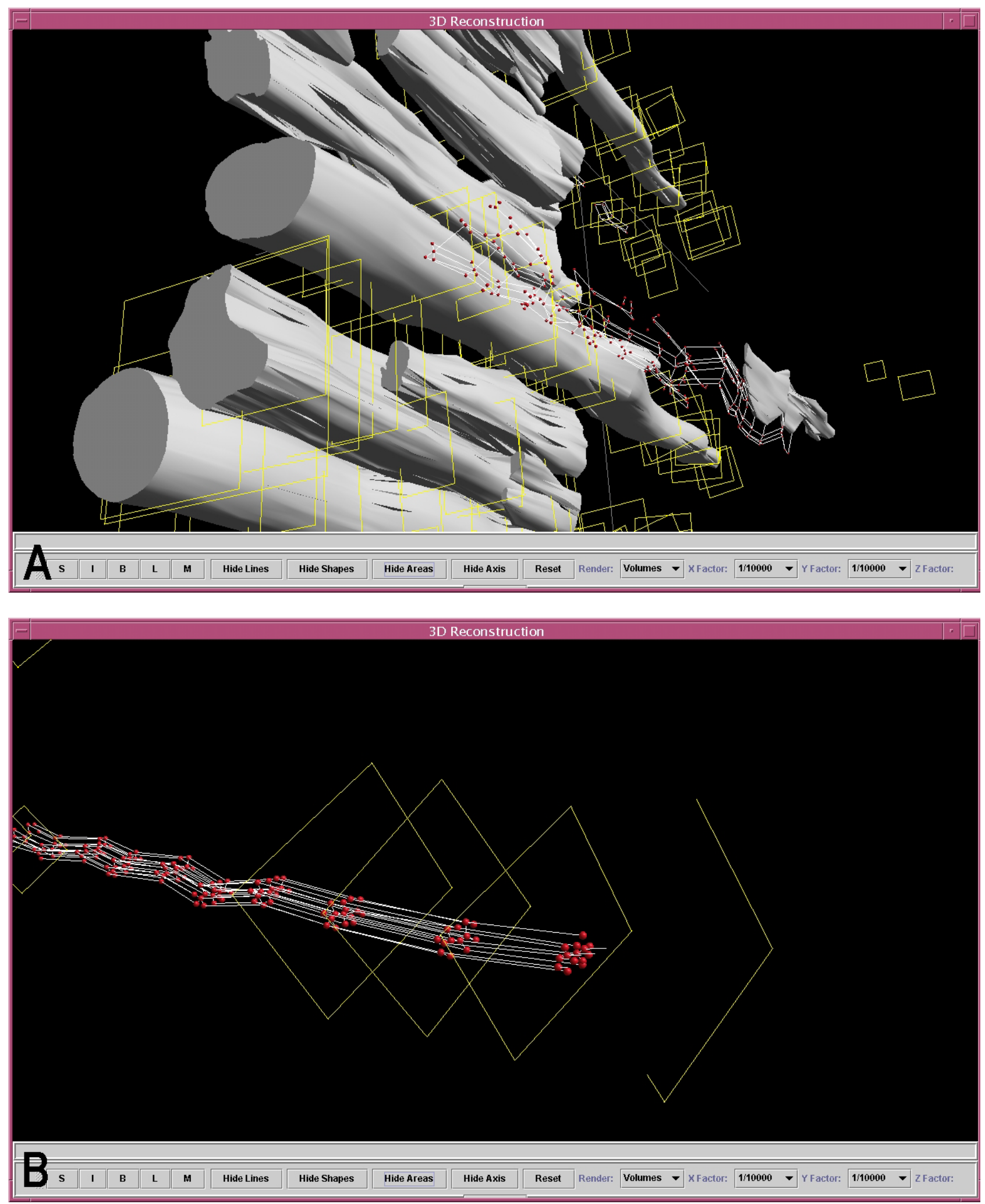
Figure 8

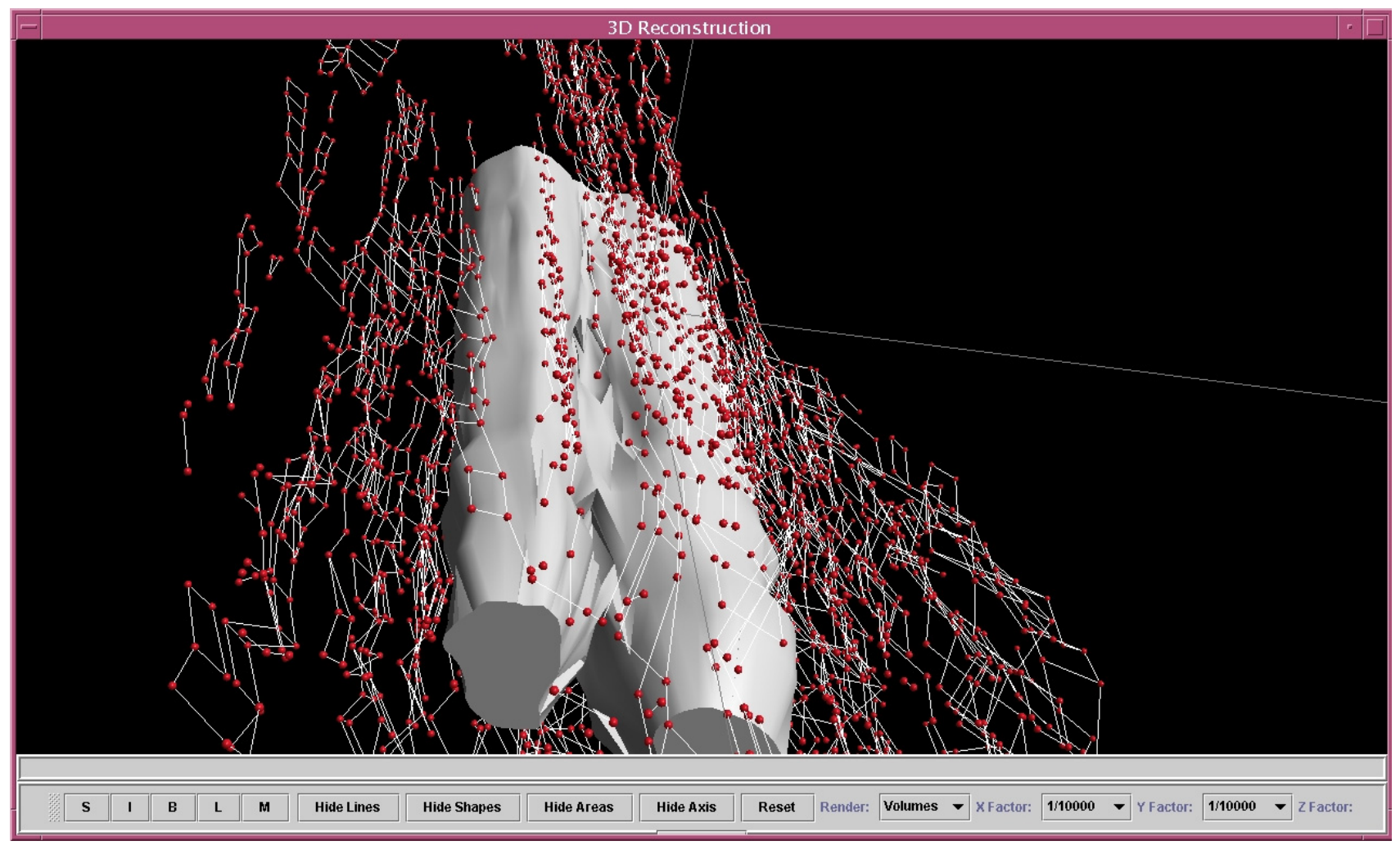


Figure 9
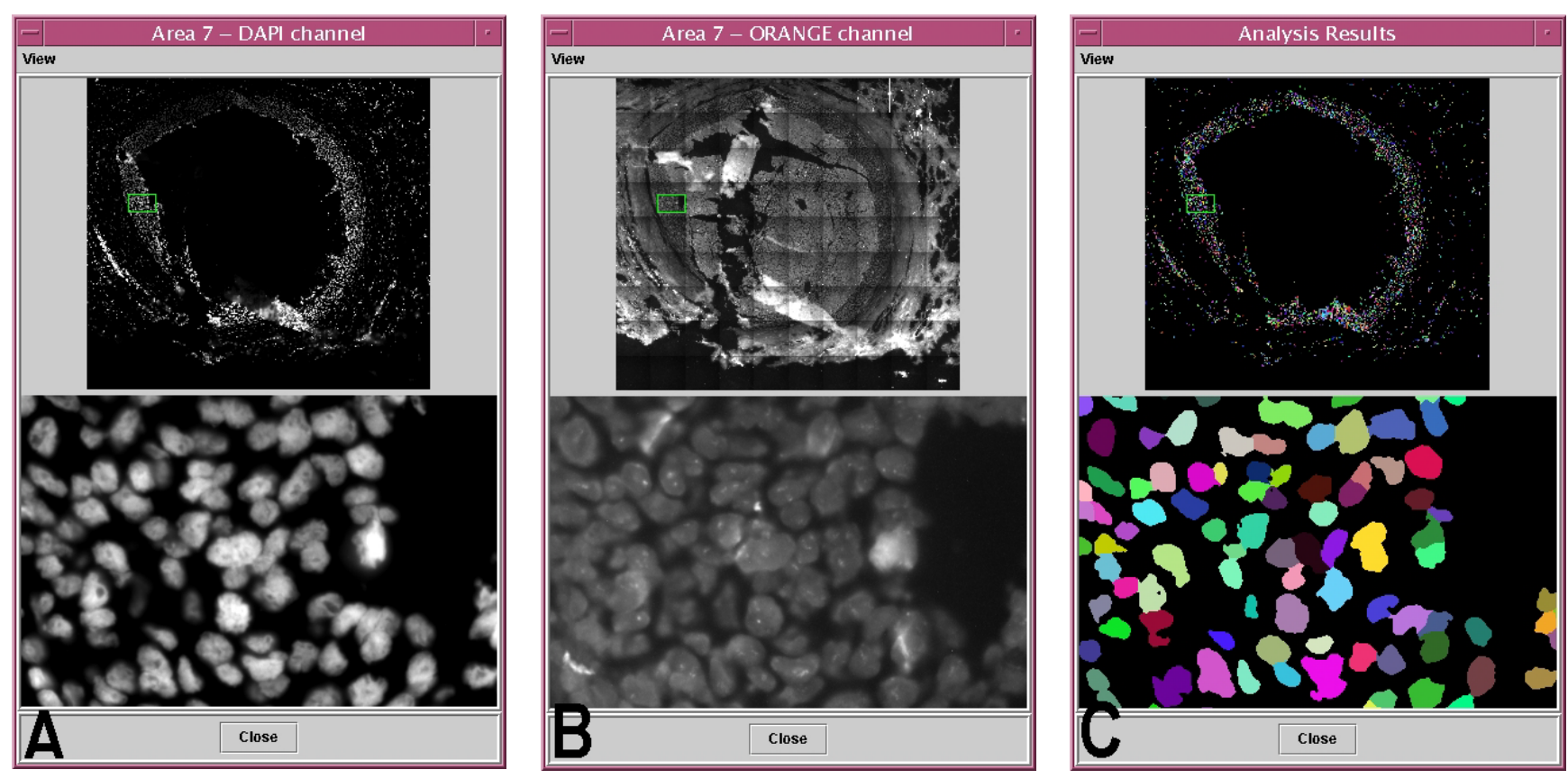